\title{
Type 1 diabetes in adults in the UK: still a displaced population right under our noses?
}

\author{
HELEN HOPKINSON
}

\section{Introduction}

Mirroring European politics, the world of type 1 diabetes care for adults in the UK appears still to be disorganised. Personal and organisational egos flex to and fro over the fraught issue of how to train people to use insulin effectively. Why can't we pull together a coherent plan? How have secondary care specialists lost their directional role in the type 1 diabetes pathway? In an increasingly complex area of therapy and technology, can we justify the transfer of our specialist workload in type 1 diabetes to our primary care colleagues? We seem to be watching as boatloads of our adults with type 1 diabetes drown in the ancient ocean of twice daily mixed insulin, and flounder in the Mediterranean sea of fixed dose multiple daily injections.

Our colleagues in the charity sector of the diabetes world exhort us to build a strategy, redesign services, commission the gold-standard care pathway; and they support us valiantly in our cries that something must be done - a situation mirrored again in Europe as the millions of refugees fleeing war are helped and cared for by the charities that do so much good work. Why are we not succeeding?

The acute issues of asylum seekers in European countries are laid before us daily by the media. We can see them and hear their pain; just as we in our diabetes clinics are faced with thousands of adults with type 1 diabetes who have never had any training in insulin dose adjustment or carbohydrate counting, and are suffering from hypoglycaemia and preventable complications. The lifestyle and eating habits (and perhaps jobs and relationships) of these people have been more regimented than necessary for years, and often their metabolic control has been suboptimal. In the diabetes world they are the obvious pressing priority and their needs must be addressed. However, it is surely just as important to attempt to stem the flow? Adults with newly diagnosed type 1 diabetes must be trained in a new way so that they are never faced with the daunting task of learning to live a new life after many years of restriction and suboptimal metabolic control. The skills required from the start include carbohydrate counting for prandial insulin adjustment as part of a free diet; basal insulin adjustment; and strategies for sport, illness, exertion, drugs, sex and

Victoria Ambulatory Care Hospital, Glasgow, UK

Address for correspondence: Dr Helen Hopkinson Consultant Physician, Diabetes Department, Victoria Ambulatory Care Hospital, Grange Road, Glasgow G42 9LF, UK

Tel: 01414516169

E-mail: helen.hopkinson@nhs.net

Br J Diabetes 2016;16:50-51

http://dx.doi.org/10.15277/bjd.2016.075 alcohol. Our colleagues in paediatric diabetes have achieved this change in attitude - why haven't we all in the adult service?

Solving the problem of political refugees in Europe is outwith our collective power, but surely improving the pathway for type 1 diabetes in adults is not? In the diabetes market place, constant posturing and competitive data production relating to multiple type 1 diabetes education packages are clouding our view of the bigger issue. They all have different curricula and terminology, and only a few have an evidence base of efficacy or cost-effectiveness. People need consistency in philosophy and principles, preferably consistency in language, and definite consistency in support. We should aim to steer the newly diagnosed into the gold-standard skill set from day 1 , and at the same time look at short brief interventions to help the long-diagnosed understand that there may be new ideas that can help them, before steering them towards a gold-standard programme when they are ready to change. We must also strive to improve our gold standards with ongoing high-quality research and development.

There is more than one thing going on here, and we must not allow three distinct issues to become confused in the minds of our users, nor of the commissioners and government.

- In line with the new NICE guideline update, the newly diagnosed adult with type 1 diabetes should be provided with carbohydrate counting training and prandial insulin adjustment skills from day 1; progression to a gold-standard training course for intensive insulin management should be a rite of passage towards the end of the honeymoon phase (when endogenous insulin production ceases).

- The long-diagnosed adult with type 1 diabetes whose metabolic control is not at target should be made aware of the evidencebased training available. It should be in every Clinical Commissioning Group and Health Board: if not ready to embark immediately on a gold-standard training course, a brief intervention should be offered to develop 'carbohydrate awareness' until a gold-standard training programme can be attempted.

- The long-diagnosed adult with type 1 diabetes who is already enlightened about new training strategies available should gain access to a gold-standard programme immediately, subject to national waiting time standards. Waiting times of 2 years or more are unacceptable and reinforce the notion that this is an optional luxury extra.

Just as government must engage with the horrors triggered by our neighbours fleeing from war, so must type 1 diabetes specialists evolve a national strategy to tackle the displacement crisis amongst adults with type 1 diabetes. I believe many amongst the diabetes healthcare population are ready to be radical and redesign services; 
but if we are to achieve the quality, consistency and sustainability our users deserve, we must become united nationally and be supported by a robust central structure that is prepared to demand and support the highest standards for our people.

I make no apologies for appealing to the type 1 diabetes community to unite. As Chair of the Dose Adjustment For Normal Eating (DAFNE) Executive Board, I am perhaps more aware than most of the tremendous difficulties faced by local diabetes teams in securing changes in their local type 1 pathway and encouraging less enthusiastic colleagues to embark on new training or work in different ways. However, my personal experience in Glasgow is that starting with one small team of consultant, diabetes specialist nurse and dietitian can quickly generate an unstoppable wave of enthusiasm, often driven by the user population. The experience of a patient asking to move to another clinic or a direct question from a patient wanting to know why she can't access the training that her neighbour has been offered are far more powerful drivers of change than peer persuasion or another business planning meeting. Get your users to come to the commissioning meetings. The DAFNE collaborative has an active User Action Group representing a network covering the whole UK who would be pleased to provide a member willing to deliver powerful first-hand testimony of their user experience.

Our experience in Glasgow has led to the development of a DAFNE service for Greater Glasgow and Clyde with courses now delivered from six acute sites. Furthermore, the benefits have extended far beyond the availability of DAFNE training to a proportion of our type 1 adults. All our staff now work with shared targets, following similar algorithms and using the same diabetes-related language. This has far-reaching benefits for the whole type 1 diabetes population, whether or not they have attended a DAFNE course, and indeed spills over into benefits for the insulin-treated patient with type 2 diabetes. We have redesigned our newly diagnosed type 1 adult pathway, and I believe this is fundamental to the integration of flexible intensive insulin management to the type 1 pathway. After years of diabetes, people who undertake DAFNE training state that their life would have been so much easier if they had been shown how to use insulin this way from the start.

The DAFNE portfolio is constantly evolving (www.dafne.uk.com). There is a DAFNE course for current pump users which is ideal for young adults who have progressed through transition but need the education that their parents had when they were a child, as well as pump users needing an update. There is a 5-week DAFNE format which suits both workers who cannot take a whole week off work and individuals for whom a slower pace of learning may be more suitable, such as older people. The 5-week format is also convenient for less than full-time healthcare professionals to deliver, and causes less disruption to the clinic schedule in small clinical teams. Training materials are now available online for participating services to enable them to deliver and quality assure all healthcare professionals who need DAFNE awareness skills but are not delivering courses.

We all know what we need to do, and the NICE update spells it out very plainly. Why try to reinvent the wheel when there is an offthe-shelf solution that can fulfil all the requirements together with the additional benefits of quality assurance, staff training and Continuing Professional Development, regular updates, and optional research involvement? We cannot afford not to do it. A DAFNE course costs around $f 50$ per patient in a service delivering one DAFNE course a month. Annual cost savings as verified by QIPP 2013 on the NHS evidence base are over $£ 93,000$ per 100,000 population every year ( $₫ 48$ million nationally, annually) with upfront training and resource costs of $£ 6,285$ to train a team of one doctor and four educators.

Support for teams contemplating setting up a DAFNE service is available from dafne@northumbria-healthcare.nhs.uk

\section{Conflict of interest None \\ Funding None}

